\title{
Validation of Affinity Differences between RNA Aptamers and a Target Protein using MALDI-TOF MS
}

\author{
Soomi Ju, Hyun-Mi Kwon, Dong-Eun Kim, and Woon-Seok Yeo* \\ Department of Bioscience and Biotechnology, Konkuk University, Seoul 143-701, Korea. *E-mail: wsyeo@konkuk.ac.kr \\ Received June 27, 2011, Accepted July 6, 2011
}

Key Words : Aptamers, Helicase, MALDI-TOF MS, Self-assembled monolayers

Aptamers are oligonucleotides or peptide molecules that bind to a specific target molecule with high affinity. In particular, nucleic acid aptamers can be selected among randomized sequences using the Systematic Evolution of Ligands by Exponential enrichment (SELEX) method, in which selections of oligonucleotides that bind to the target molecule are iterated. ${ }^{1,2}$ Thus, selection of nucleic acid aptamers with a high affinity entails a legitimate assay to differentiate affinities among aptamer candidates. Several affinity assay methods have been adapted to isolate the nucleic acid aptamer that selectively binds to the target protein, including an electrophoretic mobility shift assay, nitrocellulose filter binding assay, and affinity chromatography. ${ }^{3}$ For example, the nitrocellulose filter binding assay utilizes a property of the nitrocellulose filter that retains only oligonucleotides that are bound to the protein., ${ }^{4,5}$ Unbound oligonucleotides pass through the nitrocellulose filter without retention onto the filter membrane. However, in general, these methods require moderate amounts of the target protein, as well as a procedure for labeling oligonucleotides for discernment of signals from background reading.

In this communication, we report on a method for simple validation of affinity difference between candidate RNA aptamers obtained through screening, thus, facilitating optimum aptamer determination by combination of aptamerpresenting chips and matrix assisted laser desorption/ ionization time of flight mass spectrometry (MALDI-TOF MS). As a model system, RNA aptamers against Severe Acute Respiratory Syndrome (SARS) coronavirus helicase (SCV helicase) were employed. SARS coronavirus, which claimed almost 800 lives in southern China within a period of a few months between 2002 and 2003, is a serious concern worldwide due to absence of a vaccine or effective drug. The SARS viral genome encodes a number of nonstructural proteins (nsPs), which are indispensible for viral RNA genome replication. ${ }^{6,7}$ Among these, nsP13 harboring RNA helicase activity was suggested as an attractive target for development of an anti viral agent. ${ }^{8}$ We previously isolated inhibitory RNA aptamer pools that bind to SCV helicase (nsP13), which showed efficient inhibition of viral helicase activity for unwinding duplex DNA. ${ }^{9}$

Our strategy utilized self-assembled monolayers (SAMs) on gold to prepare aptamer-presenting chips. Figure 1(a) shows the structure of the monolayer and chemical reactions taking place on the surface. The carboxylic acid-presenting monolayer which was prepared with tri(ethylene glycol)terminated alkanethiol and acid penta(ethylene glycol)terminated alkanethiol in a ratio of 10:90, was coupled to $\mathrm{N}$ aminoethyl maleimide in order to introduce thiol groups. Thiolated anchor DNA was then conjugated to the resulting maleimide by way of the Michael addition. ${ }^{10}$ Surrounding tri(ethylene glycol) groups provide inertness to nonspecific adsorption of proteins which is the most demanding feature
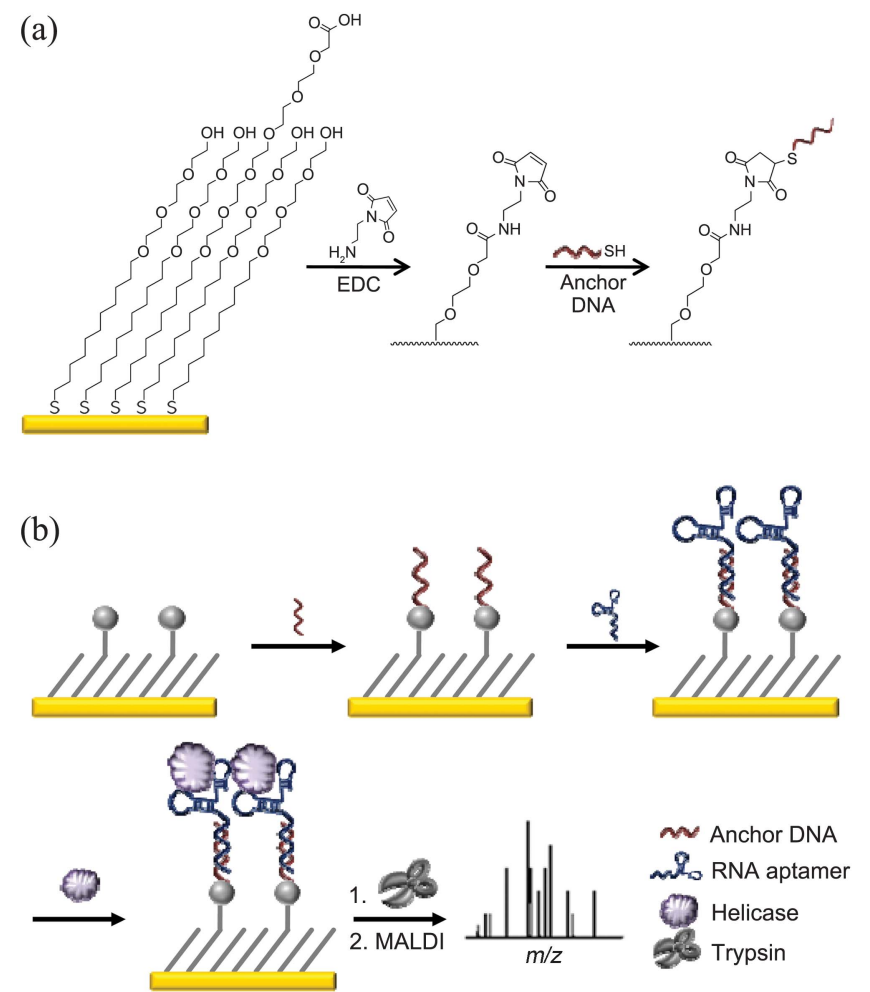

Figure 1. Schematic presentation of the strategy for validation of affinity differences between RNA aptamers and target protein. (a) Structure of SAMs on gold and chemical modifications to prepare RNA aptamer-presenting biochips. The carboxylic acid-presenting monolayer is coupled to $N$-aminoethyl maleimide, followed by thiolated anchor DNA. (b) Anchor DNA is immobilized on a maleimide-presenting monolayer, followed by hybridization of RNA aptamers on the resulting anchor DNA-presenting monolayer. Helicase is captured on the RNA aptamer-presenting monolayer, followed subsequently by digestion by trypsin and analysis by MALDI-TOF MS. 
of surfaces for interrogation of specific interactions between biomolecules at interfaces. ${ }^{11}$ Chemical conversions on the monolayer were established by direct MALDI-TOF analysis of SAMs, termed SAMDI, ${ }^{12}$ as depicted in Figure 1S (see supporting information). SAMDI analyses ensured that the chemical conversions proceeded as shown in Figure 1(a), in high yield. Figure 1(b) describes the strategy for validation of affinity differences between RNA aptamers and target protein. Following introduction of anchor DNA to maleimide-presenting monolayers, RNA aptamers are hybridized on the resulting anchor DNA-presenting monolayers. Helicase is captured on the RNA aptamer-presenting monolayer and subsequently digested by trypsin and analyzed by MALDI-TOF MS. Comparison of peak intensities of the MS spectra for each aptamer-presenting monolayer allows for quick discrimination between strong and weak affinity of aptamers toward the target protein.

First, we performed a nitrocellulose filter binding assay to measure binding affinities of six RNA aptamers that were previously selected against SCV helicase. ${ }^{9}$ The dephospho- ylated RNA aptamers were end-labeled with $\left[\gamma_{-}{ }^{32} \mathrm{P}\right] \mathrm{ATP}$ using T4 polynucleotide kinase. Each ${ }^{32} \mathrm{P}$-labeled RNA aptamer was incubated with SCV helicase, and the reaction mixture was filtered through a nitrocellulose membrane. After exposure of the membrane for visualization of radioactive RNA aptamers bound to the protein, radioactivity was quantified and normalized as shown in Figure 2(a).

Next, by combination of aptamer-presenting biochips and MALDI-TOF MS, binding affinity differences were validated between two RNA aptamers, ES15-5 and ES15-6 (Figure 2(b)), which were selected for comparison of the highest and the lowest affinities according to filter binding assay. For immobilization of aptamers to the monolayer, 3' ends of aptamers were modified with additional 23 mer oligonucleotides which was complementary to anchor DNA (for full sequences of aptamers and anchor DNA used in this study, see Table 1S in supporting information). Anchor DNApresenting monolayers prepared as described above were incubated with RNA aptamers and the resulting RNA aptamer-presenting monolayers were treated with SCV (a)

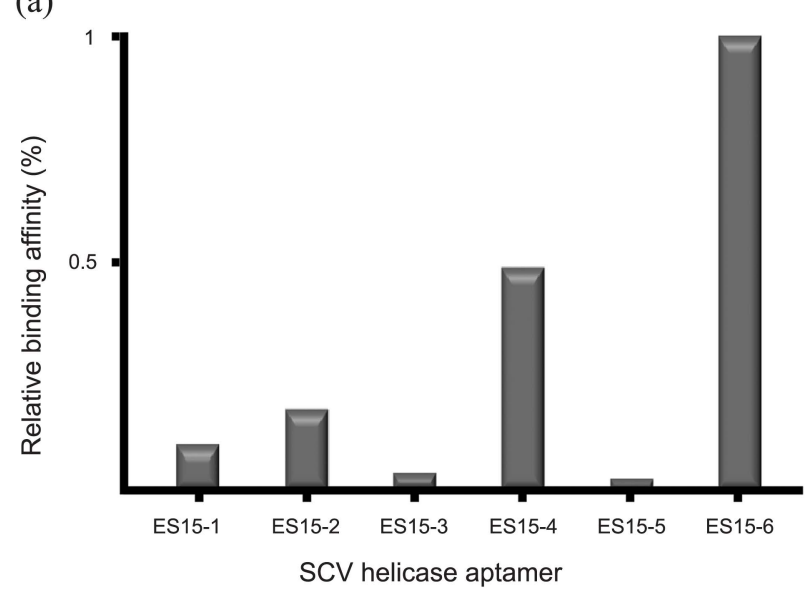

(c) (b)
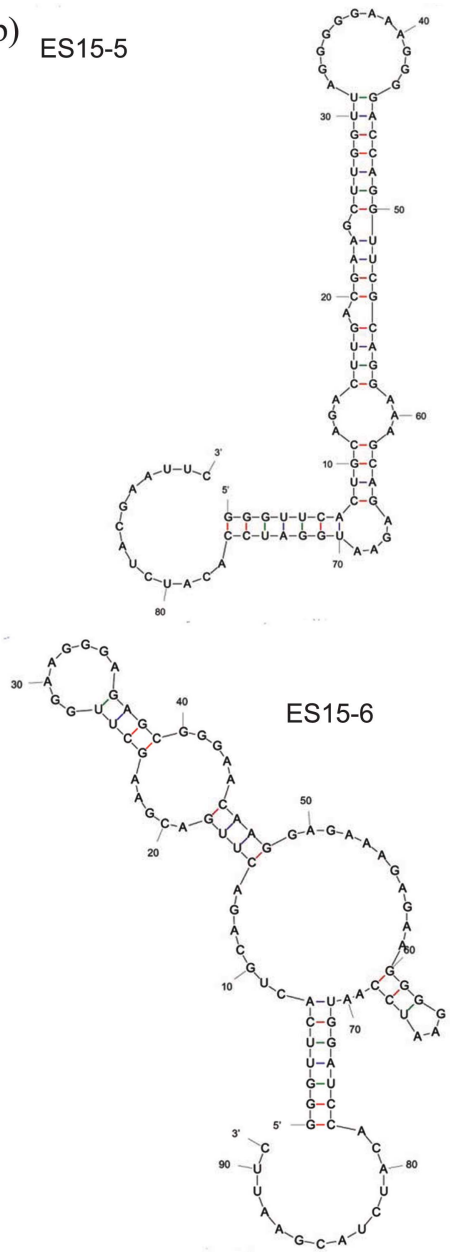

Figure 2. Binding affinities of RNA aptamers to SCV helicase and validation by MALDI-TOF MS. (a) Binding affinities of RNA aptamers to SCV helicase obtained by a nitrocellulose filter binding assay. Each ${ }^{32} \mathrm{P}-$ labeled RNA aptamer was incubated with SCV helicase, and the reaction mixture was filtered through a nitrocellulose membrane. Following exposure of the membrane for visualization of radioactive RNA aptamers bound to the protein, radioactivity was quantified and normalized. (b) Secondary structures of two RNA aptamers, ES15-5 and ES15-6, selected for comparison of the highest and the lowest affinities. C) Validation of binding affinity differences using MALDI-TOF MS. On-chip digestion of helicase captured on the ES15-6 aptamer-presenting monolayer (top), captured on the ES15-5 aptamer-presenting monolayer (middle), and captured on the anchor DNA-presenting monolayer, i.e. no aptamer present (bottom). 
helicase, followed by on-chip digestion with trypsin. The monolayers were then analyzed by MALDI-TOF MS using $\alpha$-cyano-4-hydroxycinnamic acid as a matrix. MS analysis of the ES15-6 aptamer-presenting monolayer gave several distinctive peaks corresponding to peptides fragments of helicase (Figure 2(c), top), while the ES15-5 aptamer-presenting monolayer only afforded traces (Figure 2(c), middle). As a control, we carried out the same experimental procedure using the anchor DNA-presenting monolayer, except for a helicase treatment step. As expected, no apparent MS peaks were observed (Figure 2(c), bottom). These results indicate that SCV helicase was captured on the monolayer through a specific interaction with the RNA aptamer presented on the monolayer, and furthermore, aptamer-presenting biochips and MALDI-TOF MS analysis clearly discriminated stronger binding against weaker binding to the target protein.

In general, measurement of binding affinity of selected aptamers among randomized sequences toward the target molecule is necessary for determination of an optimum aptamer with the strongest binding affinity. However, performing measurements for all of the aptamers is tedious, time-consuming, and sometimes demands significant amounts of materials. In this context, a simple validation method for discrimination between stronger binding and weaker binding would facilitate optimum aptamer determination. We believe that our strategy for simple validation of affinity differences between candidate RNA aptamers by combination of aptamerpresenting chips and MALDI-TOF MS is well suited for this purpose. In addition, use of SAMs of alkanethiolates on gold is advantageous over other types of solid substrates. SAMs on gold provide inertness toward non-specific protein adsorption and compatibility with existing conjugation chemistry for immobilization of various biomolecules with a surface density-controllable format. We believe that the current study described here should be extendable to other types of screening, such as protein-protein, and proteinsmall molecule interactions.

\section{Experimental Section}

Preparation of Carboxylic Acid-presenting Monolayer. The gold chips were incubated in mixed solution of tri(ethylene glycol)-terminated alkanethiol and acid penta(ethylene glycol)-terminated alkanethiol (1 $\mathrm{mM}$ in ethanol) in a ratio of 10:90 for $12 \mathrm{~h}$. The monolayers were washed with absolute ethanol and dried under a stream of nitrogen.

Preparation of Anchor DNA-presenting Monolayer. The carboxylic acid-presenting monolayer was treated with 1-ethyl-3-(3-dimethylaminopropyl) carbodiimide hydrochloride (EDC, $20 \mathrm{mg} \mathrm{mL}^{-1}$ in $\mathrm{pH} 7.4$ phosphate-buffered saline (PBS)) and $N$-aminoethyl maleimide $\left(7 \mathrm{mg} \mathrm{mL}^{-1}\right.$ in PBS) for $2 \mathrm{~h}$, washed with PBS and absolute ethanol, and dried under a stream of nitrogen. The thiolated anchor DNA (10 $\mu \mathrm{M}$ in PBS) was then covalently anchored on the maleimide-presenting monolayer by incubating for $12 \mathrm{~h}$ at $37^{\circ} \mathrm{C}$, washed with PBS and deionized water, and dried under a stream of nitrogen.

Hybridization of RNA Aptamers and Helicase Immobilization. The anchor DNA-presenting monolayers were incubated with RNA aptamers $(10 \mu \mathrm{M}$ in PBS $)$ for 30 min at $65{ }^{\circ} \mathrm{C}$ and then for $10 \mathrm{~min}$ at $4{ }^{\circ} \mathrm{C}$. The monolayers were washed with tri-distilled water and PBS, and dried under a stream of nitrogen. The resulting RNA aptamerpresenting monolayers were treated with helicase $(5.5 \mu \mathrm{M}$ in PBS) for $1 \mathrm{~h}$, rinsed with PBS and tri-distilled water, dried under a stream of nitrogen, and stored at $4{ }^{\circ} \mathrm{C}$ until use.

On-chip Digestion. The $5 \mu \mathrm{L}$ of trypsin solution (200 ng $\mu \mathrm{L}^{-1}$ in $20 \mathrm{mM}$ ammonium bicarbonate, $0.5 \mathrm{mM}$ calcium chloride, and $10 \%$ acetonitrile) was applied to the helicasebound monolayers and incubated at $37^{\circ} \mathrm{C}$ for $6 \mathrm{~h}$, and then the monolayers were dried at ambient condition. The monolayer was analyzed by MALDI-TOF MS, using the 1 $\mu \mathrm{L}$ of $\alpha$-cyano-4-hydroxycinnamic acid (CHCA, $1 \mathrm{mg} 150$ $\mu \mathrm{L}^{-1}$ in $50 \%$ acetonitrile, $0.05 \%$ TFA in distilled water) as a matrix.

Analysis of MALDI-TOF MS. Mass analysis was performed using an Autoflex III MALDI-TOF mass spectrometer (Bruker Daltonics). All of the spectra were acquired with $19 \mathrm{kV}$ accelerating voltage, a $50 \mathrm{~Hz}$ repetition rate, and positive mode with the average of $\sim 700$ shots.

Acknowledgments. This research was supported by Basic Science Research Program through the National Research Foundation of Korea (NRF) funded by the Ministry of Education, Science and Technology (grant 2010-0021191 and 2010-0019306).

\section{References}

1. Ellington, A. D.; Szostak, J. W. Nature 1990, 346, 818.

2. Tuerk, C.; Gold, L. Science 1990, 249, 505.

3. Vortler, C. S.; Milovnikova, M. Wiley-VCH Verlag GmbH \& Co. 2005.

4. Soderman, K.; Reichard, P. Anal. Biochem. 1986, 152, 89.

5. Oehler, S.; Alex, R.; Barker, A. Anal. Biochem. 1999, 286, 330.

6. Lee, N. R.; Kwon, H. M.; Park, K.; Oh, S.; Jeong, Y. J.; Kim, D. E. Nucleic Acids Res. 2010, 38, 7626.

7. Ivanov, K. A.; Thiel, V.; Dobbe, J. C.; van der Meer, Y.; Snijder, E. J.; Ziebuhr, J. J. Virol. 2004, 78, 5619.

8. Tanner, J. A.; Watt, R. M.; Chai, Y. B.; Lu, L. Y.; Lin, M. C.; Peiris, J. S.; Poon, L. L.; Kung, H. F.; Huang, J. D. J. Biol. Chem. 2003, 278, 39578.

9. Jang, K. J.; Lee, N. R.; Yeo, W. S.; Jeong, Y. J.; Kim, D. E. Biochem. Biophys. Res. Commun. 2008, 366, 738.

10. Lee, J. R.; Lee, J.; Kim, S. K.; Kim, K. P.; Park, H. S.; Yeo, W.-S. Angew. Chem. Int. Ed. 2008, 47, 9518.

11. Mrksich, M.; Whitesides, G. M. ACS Symp. Ser. 1997, 680, 361.

12. Mrksich, M. ACS Nano. 2008, 2, 7. 